\title{
Study of the solar modulation for the cosmic ray isotopes with the PAMELA experiment
}

\author{
Alex Lenni, ${ }^{a, b, c, *}$ M. Boezio, ${ }^{b, c}$ R. Munini, ${ }^{b, c}$ W. Menn, ${ }^{d}$ N.Marcelli, ${ }^{e, f}$ M. S. Potgieter, ${ }^{g}$ \\ D. Bisschoff, ${ }^{h}$ M. D. Ngobeni ${ }^{h}$ and O. P. M. Aslam ${ }^{h}$ on behalf of the PAMELA
}

\section{Collaboration}

(a complete list of authors can be found at the end of the proceedings)

${ }^{a}$ Department of Physics, University of Trieste, I-34127 Trieste, Italy

${ }^{b}$ INFN, Section of Trieste, I-34127 Trieste, Italy

${ }^{c}$ IFPU - Institute of Fundamental Physics of the Universe, I-34151 Trieste, Italy

${ }^{d}$ Department of Physics, University of Siegen, D-57068 Siegen, Germany

${ }^{e}$ Department of Physics, University of Rome Tor Vergata, I-00133 Rome, Italy

${ }^{f}$ INFN, Section of Rome Tor Vergata, I-00133 Rome, Italy

${ }^{g}$ Institute for Experimental and Applied Physics, University of Kiel, D-24118, Kiel, Germany

${ }^{h}$ Centre for Space Research, North-West University, 2520, Potchefstroom, South Africa

E-mail: alex.lenni@ts.infn.it, riccardo.munini@ts.infn.it,

mirko.boezio@ts.infn.it, menn@pamela.physik.uni-siegen.de, nadir.marcelli@roma2.infn.it, marius.s.potgieter@gmail.com, driaanb@gmail.com, donald.ngobeni@nwu.ac.za, aslamklr2003@gmail.com

\footnotetext{
${ }^{*}$ Presenter
} 
The space-borne PAMELA experiment was launched on the 15th June 2006 on board the Russian satellite Resurs-DK1 from the Baikonur cosmodrome. PAMELA performed high-precision measurements of cosmic rays over a wide energy range until January 2016. Owing to its long-duration operation, PAMELA had turned out to be an optimal detecting apparatus for studies of the solar modulation of cosmic rays over time. The PAMELA collaboration has already published timedependent proton, helium and electron spectra as well as the positron to electron ratio spanning almost an entire solar cycle. These results are fundamentally important in the fine-tuning of propagation and modulation models of cosmic rays through the Heliosphere.

In this talk, the yearly average spectra for protons, deuterons, Helium-3 and Helium-4 nuclei are presented for the 23rd solar minimum (July 2006 - January 2009) and the first part of the 24th solar maximum (until September 2014). The isotopic composition was measured between 0.1 and $1.1 \mathrm{GeV} / \mathrm{n}$ using two different detector systems. As expected, the measured spectra display a rising trend towards the solar minimum followed by a decreasing trend as the solar maximum approaches. The time-dependent ratio of these isotopes is also presented. According to solar modulation studies, a non-constant ratio is expected due to the different charge-to-mass ratios and the different shapes of the respective local interstellar spectra.

Additionally, it is of interest to analyze the observed spectra and ratios with state-of-the-art solar modulation models to obtain a deeper understanding of the relative importance of the mechanisms responsible for the propagation of cosmic rays in the Heliosphere over time.

$37^{\text {th }}$ International Cosmic Ray Conference (ICRC 2021)

July 12th-23rd, 2021

Online - Berlin, Germany 


\section{Introduction}

The ${ }^{2} \mathrm{H}$ and ${ }^{3} \mathrm{He}$ isotopes in cosmic rays (CRs) have mostly a secondary origin, resulting from the nuclear interactions of primary $\mathrm{CR}$ protons and ${ }^{4} \mathrm{He}$ with the interstellar medium (ISM). The spectral shape and composition of the secondary isotope are therefore completely determined by the spectrum of the primary parent elements, by the related production cross sections and by the propagation process. Measurements of the secondary isotopes spectra are then a powerful tool to constrain the parameters of the galactic propagation models. The PAMELA collaboration already published results on the ${ }^{1} \mathrm{H},{ }^{2} \mathrm{H},{ }^{3} \mathrm{He}$ and ${ }^{4} \mathrm{He}$ isotopes between $\sim 0.1$ and $1.1 \mathrm{GeV} / \mathrm{n}$ with data collected between July 2006 and December 2007 [1].

Below few tens of $\mathrm{GeV} / \mathrm{n}, \mathrm{CRs}$ are heavily affected by the solar modulation, which reduces their intensity and modifies their spectral shape with respect to the Local Interstellar Spectrum (LIS), i.e. the spectrum of CRs outside the Heliosphere. Moreover, the solar modulation introduces a time dependence in the CR fluxes measured inside the Heliosphere. During solar maxima, the CR intensity is lower with respect to periods of solar minima as a consequence of the increased solar activity, which induces a more turbulent solar wind, a stronger embedded heliospheric magnetic field and a wavier current sheet. During the PAMELA operating period, the maximum CR intensity was reached around late 2009 at the end of the 23rd solar minimum while the lower CR intensity corresponds to the middle of the 24th solar maximum in 2014.

In this work, the isotope analysis has been extended to data collected with PAMELA up to September 2014 to study the time evolution of the fluxes. Precise measurements of the time dependence in the isotope spectra provide important information about their propagation mechanisms through the Heliosphere, highlighting possible different modulations caused by their mass to charge ratio and the different shapes of the respective LIS. A 3D numerical model which solves the Parker equation, e.g. see [2,3], was already calibrated and tested with the PAMELA time dependent measurements on protons $[4,5]$ and electrons [6], and is going to be used to interpret the isotope modulation.

In this contribution, the analysis performed to obtain the isotope fluxes will be discussed. To reach the best time resolution keeping good statistics, yearly average fluxes were measured. The preliminary results will be presented at the conference.

\section{The PAMELA apparatus}

The PAMELA experiment [7] was launched on the 15th June 2006 from the Baikonur cosmodrome in Kazakhstan, on board the Russian satellite Resurs DK1, and measured cosmic rays until January 2016. The PAMELA mission covers almost an entire solar cycle, from the middle of the 23rd solar minimum to the middle of the 24th solar maximum. Hence, the data collected during this time represent an excellent chance to study the time-dependent evolution of the low energy cosmic radiation due to solar modulation.

A schematic view of the PAMELA experiment is shown in Figure 1. The core of the apparatus is a magnetic cavity equipped with six planes of double face microstrip silicon detectors. The tracker is used to measure the rigidity $R$ of the incoming particle defined as $R=\frac{p c}{Z e}$, where $p$ is the particle momentum, $c$ the speed of light and $Z e$ the electric charge. Besides, the tracker planes provide 


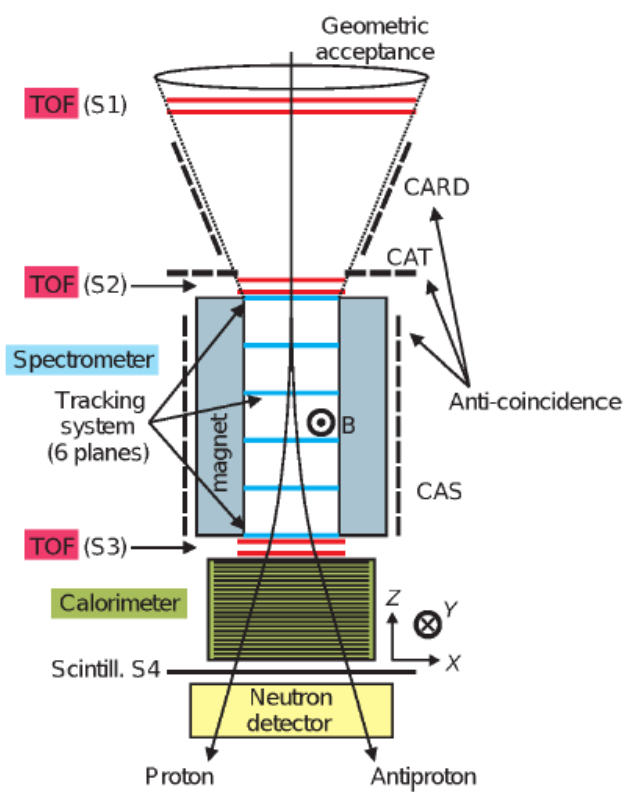

Figure 1: Schematic view of the PAMELA apparatus.

twelve independent energy loss measurements $d E / d X$, which are extensively used in the analysis of the isotopes. A time of flight (ToF) system is composed of six layers of plastic scintillators and performs a velocity measurement, six independent $d E / d X$ measurements and trigger the data acquisition. Finally, an electromagnetic calorimeter made of 44 single-sided silicon sensor planes interleaved with 22 planes of tungsten, mainly used for hadron-lepton separation, provides multiple energy loss measurements as well. The high redundancy of the PAMELA subdetectors and the multiple independent $d E / d X$ measurements allow performing a precise study of the isotopes composition of CR hydrogen and helium.

\section{Data analysis}

Two different analyses were developed, one for the separation of the hydrogen isotopes and one for the separation of the helium isotopes. A few details about these two analyses are reported hereinafter.

\subsection{Event Selection}

PAMELA triggered events had to fulfill several criteria to be used for further analysis. The requirements are extensively described in [1]: the tracker was used to select a sample of events with a single track reconstructed at least $1.5 \mathrm{~mm}$ away from the magnet walls. The events must have a lever-arm of at least 4 silicon planes and a minimum of 3 hits on both the bending $x$-view and on non-bending y-view. Bad quality tracks were rejected imposing a cut on the $\chi^{2}$ of the fitting algorithm. From these events, it is then possible to extract samples of $Z=1$ and $Z=2$ particles, in which the isotopic separation can be performed. 

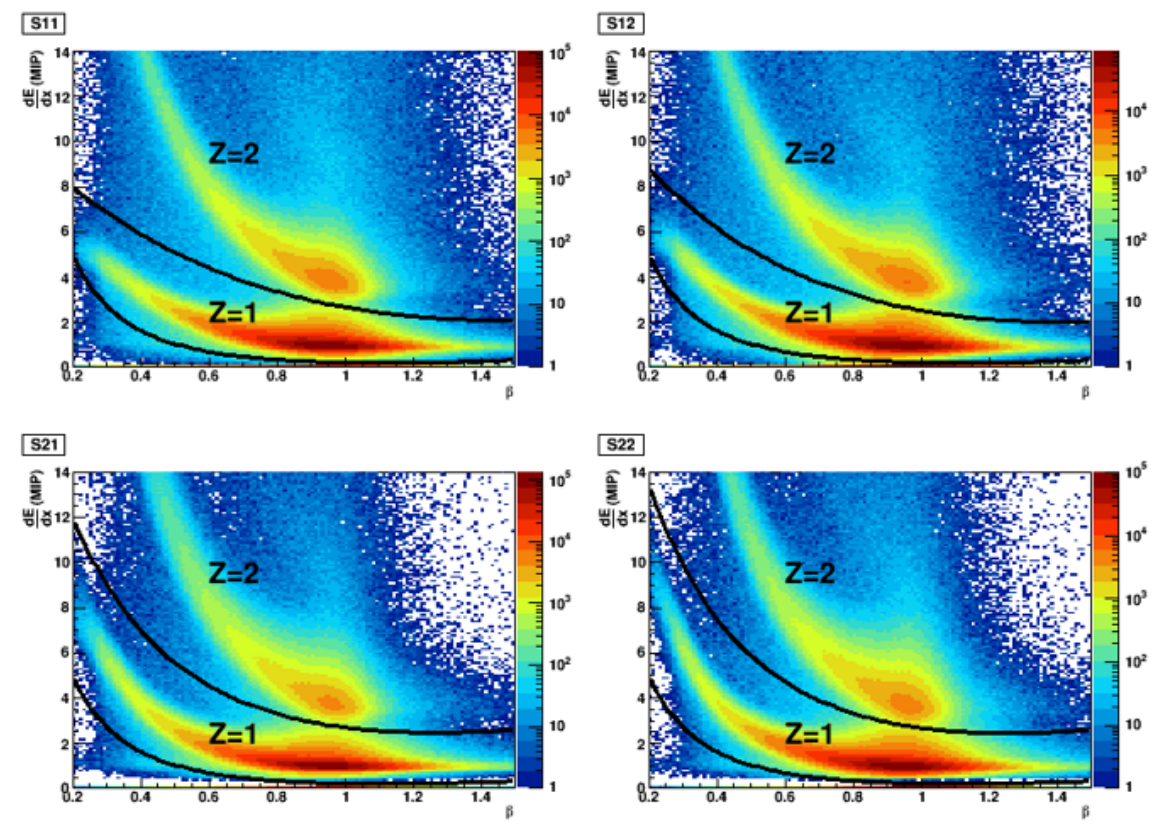

Figure 2: $d E / d X$ distributions as a function of the velocity $\beta$ for each of the first four ToF layers. The black lines represent the cuts defined to select $Z=1$ particles.

\subsection{Selection of the hydrogen isotopes}

In the hydrogen analysis, particles of electric charge $Z=1$ were selected with cuts calibrated on the $d E / d X$ distributions as a function of $\beta^{1}$, one for each of the four ToF layers above the tracker as shown in Figure 2. Moreover, these cuts allow rejecting particles with $Z=1$, resulting from the fragmentation of heavier nuclei in the detector planes above the tracker.

The isotopic separation was then performed combining the multiple $\mathrm{dE} / \mathrm{dX}$ measurements from the ToF and the tracker planes and the measured rigidity. In particular, a truncated mean to the lowest value was calculated from the tracker $d E / d X$ measurements (up to twelve), in this way those $d E / d X$ values, which typically lie on the longer Landau tail at higher values, were rejected and the resolution in $d E / d X$ distribution was improved. In Figure 3 left panel the truncated mean $d E / d X$ distribution measured in the tracker as a function of rigidity is reported for the $Z=1$ sample with the cuts calibrated to select protons and deuterons. To reject the residual but not negligible proton contamination beyond about $1.5 \mathrm{GV}$, an additional selection was needed. For this purpose, a truncated mean to the lowest value was calculated from the ToF $d E / d X$ measurements (up to six) and deuteron selection cuts were calibrated on the truncated mean ToF $d E / d X$ distribution of the events surviving the previous deuteron selection, as shown in Figure 3 right panel.

After all these selections, a clean sample of protons was obtained in the rigidity range $[0.5,2.4]$ GV. The selected deuterons instead suffer from residual proton contamination above $2.0 \mathrm{GV}$. In order to overcome this problem, a fit of the $1 / \beta$ distributions with two Gaussians, one for protons and one for deuterons, was performed, allowing to estimate the number of selected deuterons with negligible residual contamination.

${ }^{1} \beta=v / c$ with $v$ the velocity of the particle and $c$ the speed of light in vacuum 

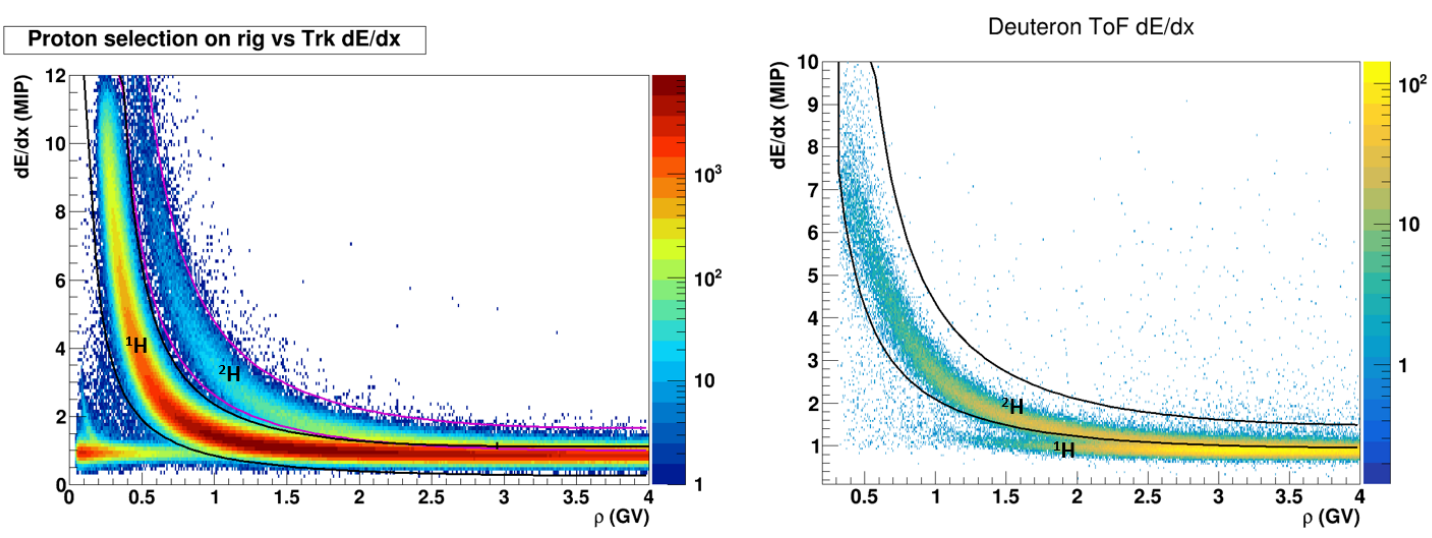

Figure 3: Left panel: The distribution of the truncated mean $d E / d X$ measured in the tracker as a function of rigidity for $\mathrm{Z}=1$ particles. The black lines represent the proton cut and the violet lines represent the deuteron cut. Right panel: The distribution of the truncated mean $d E / d X$ measured in the ToF system as a function of the rigidity for the deuteron sample selected with the tracker $d E / d X$ selection. The black lines represent the deuteron cut in $\mathrm{ToF} d E / d X$.
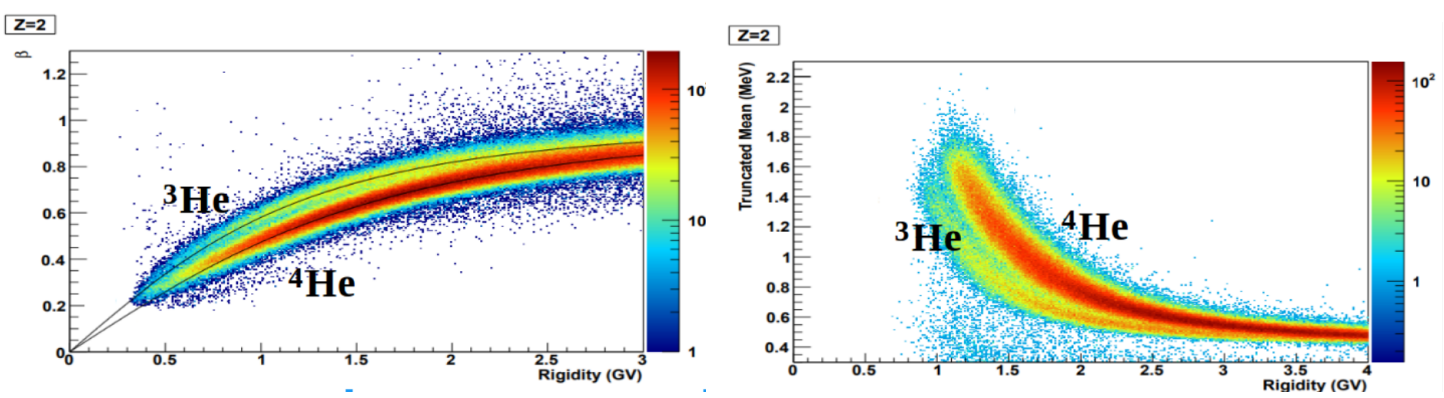

Figure 4: Left panel: $\beta$ distribution as a function of the rigidity for $Z=2$ particles. The black lines represent the expected theoretical $\beta$ values for the two different isotopes. Right panel: The distribution of the truncated mean $d E / d X$ measured in the calorimeter as a function of the rigidity for $Z=2$ particles.

\subsection{Selection of the helium isotopes}

In the helium analysis, a sample of particles with charge $Z=2$ was selected on the average $d E / d X$ distribution measured in the tracker as a function of the rigidity. From this sample, the isotopic separation was performed implementing two different techniques:

- Isotope separation using the rigidity versus $\beta$ distribution. Figure 4 left panel shows the $\beta$ distribution as a function of rigidity for $Z=2$ particles selected with the tracker. The black lines in the figure represent the theoretical curves for each isotope.

- Isotopic separation with multiple $\mathrm{dE} / \mathrm{dX}$ in the calorimeter as a function of rigidity for noninteracting events. To select non-interacting particles in the calorimeter, the total energy detected $q_{t o t}$ and the energy deposited in the strip closest to the track and in the neighboring strip on each side, $q_{\text {track }}$, were derived for each silicon layer. Starting from the top of the calorimeter the $\sum q_{\text {track }} / \sum q_{\text {tot }}$ was calculated down to the last layer and the events with $\sum q_{\text {track }} / \sum q_{\text {tot }}>0.9$ were recognised and selected as non-interacting. This 0.9 value was 


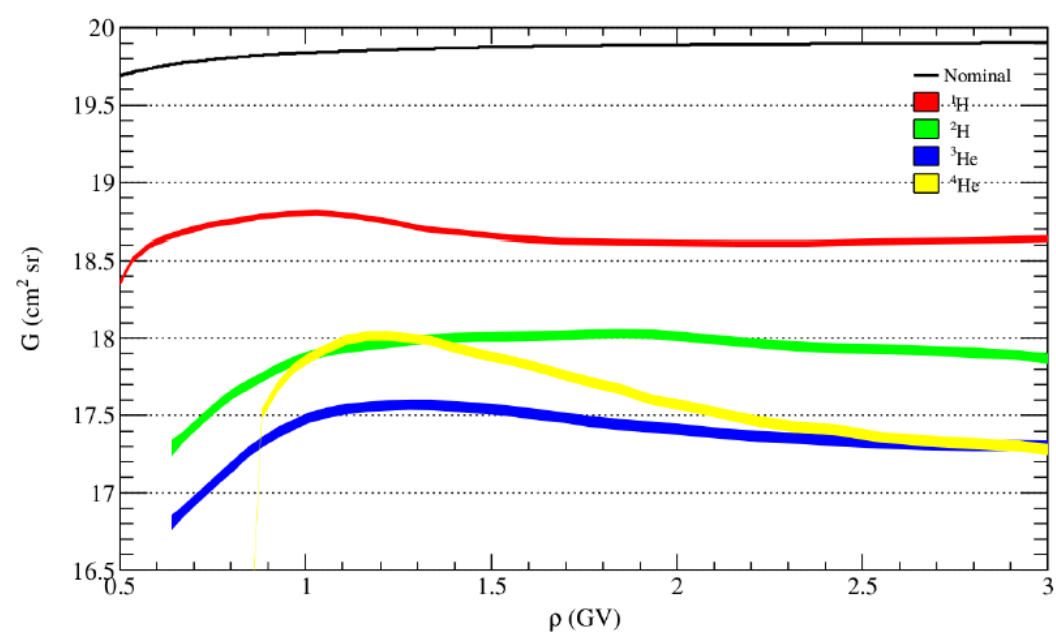

Figure 5: Nominal geometrical factor $G(E)$ (black line) and the effective geometrical factor $G$ for the four different isotopes under study as a function of rigidity.

chosen since it was found to give a good compromise between high efficiency and rejection of interactions. For these non-interacting events, a truncated mean was calculated as the average of the half of the calorimeter $\mathrm{dE} / \mathrm{dX}$ measurements with the lower values, requiring a minimum of at least $5 \mathrm{dE} / \mathrm{dX}$ measurements used for the mean calculation. This approach made possible to reduce the effect of the Landau tails and consequently enhance the resolution. Figure 4 right panel shows the resulting truncated mean $d E / d X$ as a function of the rigidity. For more details of these selection criteria see [1].

To get the raw isotope numbers, a likelihood fit (the "TFractionFitter" method in ROOT [8]) was performed on the $1 / \beta$ distributions of the selected events in rigidity slices. The likelihood fit needs a model distribution to create the expected probability density function, the full Monte Carlo simulation of the PAMELA apparatus based on the GEANT4 was used for this task. With all these procedures it was possible to derive the numbers of events for Helium-3 and Helium- 4 in the rigidity range [1.,2.4] GV with the ToF and in $[1.5,3.0] \mathrm{GV}$ with the calorimeter.

\section{Flux calculation}

The flux $\phi(R)$ as a function of the rigidity was calculated for each of the isotopes as follows:

$$
\phi(R)=\frac{N(R)}{G(R) \cdot T \cdot \epsilon(R) \cdot \Delta R}
$$

where $N(R)$ is the number of events selected in a rigidity range of width $\Delta R$ and centered on $R, \epsilon(R)$ is the total efficiency of the selections used in the analysis, $G(R)$ is the geometrical factor and $T$ the live-time. When possible, the efficiency values were evaluated with flight data exploiting the detector redundancy to select clean samples of isotopes. Simulated data were used to cross-check the flight efficiency in order to emphasize possible contamination of the flight sample. When the flight samples resulted contaminated, the simulated efficiency was used. The tracking 
efficiency was entirely evaluated using simulated data. Figure 6 shows the nominal geometrical factor $G(R)=19.9 \mathrm{~cm}^{2} \mathrm{sr}$ (black line), which was corrected to account for the hydrogen and helium nuclei lost due to hadronic interactions in the aluminum pressurized container ( $2 \mathrm{~mm}$ thick) and in the top scintillators. Moreover, the ${ }^{2} \mathrm{H}$ and ${ }^{3} \mathrm{He}$ geometrical factor were corrected for the locally produced contamination from the fragmentation of higher nuclei in the $2 \mathrm{~mm}$ aluminum dome. These corrections were evaluated with the Monte Carlo simulation. The contribution in the ${ }^{3} \mathrm{He}$ sample from ${ }^{4} \mathrm{He}$ fragmentation was found to be less than $1 \%$ as well as for the contamination evaluated in the ${ }^{2} H$ sample. Finally, due to the finite spectrometer resolution and the ionization energy losses, the measured rigidity with the tracker system differs from the initial rigidity at the top of the payload; to overcome these effects an unfolding procedure [9] was applied.

\section{Conclusion}

The analysis of the time-dependent fluxes of the helium and hydrogen isotopes performed with the PAMELA experiment has been presented. Data acquired between July 2006 and September 2014 were used to measure the yearly average fluxes for a total of 9 spectra for each isotope. These results are very important to test and calibrate models used to describe the propagation of CR inside the Heliosphere as well as to perform a better determination of their local interstellar spectra. Preliminary results for the ${ }^{1} \mathrm{H},{ }^{2} \mathrm{H},{ }^{3} \mathrm{He}$ and ${ }^{4} \mathrm{He}$ fluxes and the related ratios are going to be presented at the ICRC oral session.

\section{References}

[1] Adriani, O., et al, ApJ, 818, 68, 2016

[2] Potgieter, Living Rev Solar Physics, 10, 3, 2013

[3] Potgieter, et al., Solar Physics, 289, 391, 2014

[4] Adriani, O., et al, ApJ, 765, 91, 2013

[5] Adriani, O., et al, ApJ Letters, 854, L2, 2018

[6] Adriani, O., et al, ApJ, 810, 142, 2015

[7] Picozza, P., et al., ApJ, 27, 296, 2007

[8] Brun, R. \& Rademakers, F, Nucl. Instrum. Meth. A 389, 81, 1997

[9] D’Agostini, G. Nuclear Instruments and Methods in Physics Research A, 362, 487, 1995 


\section{Full Authors List: PAMELA Collaboration}

O. Adriani ${ }^{1,2}$, G. C. Barbarino ${ }^{3,4}$, G. A. Bazilevskaya ${ }^{5}$, R. Bellotti ${ }^{6,7}$, M. Boezio ${ }^{8,9}$, E. A. Bogomolov ${ }^{10}$, M. Bongi ${ }^{1,2}$, V. Bonvicini ${ }^{8}$, A. Bruno $^{6}$, F. Cafagna ${ }^{7}$, D. Campana ${ }^{2}$, P. Carlson ${ }^{11}$, M. Casolino ${ }^{12,13}$, G. Castellini ${ }^{14}$, C. De Santis ${ }^{12}$, A. M. Galper ${ }^{16}$, S. V. Koldashov ${ }^{16}$, S. Koldobskiy ${ }^{16}$, A. N. Kvashnin ${ }^{10}$, A. Lenni ${ }^{8,9,17}$, A.A. Leonov ${ }^{16}$, V.V. Malakhov ${ }^{16}$, L. Marcelli ${ }^{12}$, N. Marcelli ${ }^{12,18}$, M. Martucci ${ }^{18,19}$, A. G. Mayorov ${ }^{16}$, W. Menn ${ }^{20}$, M. Merge ${ }^{12,18}$, V. V. Mikhailov ${ }^{16}$, E. Mocchiutti ${ }^{8}$, A. Monaco ${ }^{6,7}$, N. Mori $^{2}$, R. Munini ${ }^{8,9}$, G. Osteria $^{4}$, B. Panico ${ }^{4}$, P. Papini ${ }^{2}$, M. Pearce ${ }^{11}$, P. Picozza ${ }^{12,18}$, M. Ricci ${ }^{19}$, S. B. Ricciarini ${ }^{2,14}$, M. Simon ${ }^{20}$, A. Sotgiu ${ }^{12}$, R. Sparvoli ${ }^{12,18}$, P. Spillantini ${ }^{16,21}$, Y. I. Stozhkov ${ }^{5}$, A. Vacchi ${ }^{8,22}$, E. Vannuccini ${ }^{2}$, G.I. Vasilyev ${ }^{10}$, S. A. Voronov ${ }^{16}$, Y. T. Yurkin ${ }^{16}$, G. Zampa ${ }^{8}$ and N. Zampa $^{8}$

${ }^{1}$ University of Florence, Department of Physics, I-50019 Sesto Fiorentino, Florence, Italy. ${ }^{2}$ INFN, Sezione di Florence, I-50019 Sesto Fiorentino, Florence, Italy. ${ }^{3}$ University of Naples "Federico II," Department of Physics, I-80126 Naples, Italy. ${ }^{4}$ INFN, Sezione di Naples, I-80126 Naples, Italy. ${ }^{5}$ Lebedev Physical Institute, RU-119991, Moscow, Russia. ${ }^{6}$ University of Bari, Department of Physics, I-70126 Bari, Italy. ${ }^{7}$ INFN, Sezione di Bari, I-70126 Bari, Italy. ${ }^{8}$ INFN, Sezione di Trieste, I-34149 Trieste, Italy. ${ }^{9}$ IFPU - Institute of Fundamental Physics of the Universe, I-34151 Trieste, Italy. ${ }^{10}$ Ioffe Physical Technical Institute, RU-194021 St. Petersburg, Russia. ${ }^{11}$ KTH, Department of Physics, and the Oskar Klein Centre for Cosmoparticle Physics, AlbaNova University Centre, SE-10691 Stockholm, Sweden. ${ }^{12}$ INFN, Sezione di Rome "Tor Vergata," I-00133 Rome, Italy. ${ }^{13}$ RIKEN, EUSO team Global Research Cluster, Wako-shi, Saitama, Japan. ${ }^{14}$ IFAC, I-50019 Sesto Fiorentino, Florence, Italy. ${ }^{15}$ Space Science Data Center-Agenzia Spaziale Italiana, via del Politecnico, s.n.c., I-00133, Roma, Italy. ${ }^{16}$ MEPhI: National Research Nuclear University MEPhI, RU-115409, Moscow, Russia. ${ }^{17}$ University of Trieste, Department of Physics, I-34127 Trieste, Italy. ${ }^{18}$ University of Rome "Tor Vergata," Department of Physics, I-00133 Rome, Italy. ${ }^{19}$ INFN, Laboratori Nazionali di Frascati, Via Enrico Fermi 40, I-00044 Frascati, Italy. ${ }^{20}$ University of Siegen, Department of Physics, D-57068 Siegen, Germany. ${ }^{21}$ Istituto Nazionale di Astrofisica, Fosso del cavaliere 100, I-00133 Roma, Italy. ${ }^{22}$ University of Udine, Department of Mathematics, Computer Science and Physics Via delle Scienze, 206, Udine, Italy. 